\title{
Picosecond time-resolved $x$-ray diffraction probe of coherent lattice dynamics (abstract) (invited)
}

D. A. Reis, ${ }^{\text {a) }}$ M. F. DeCamp, P. H. Bucksbaum, R. Clarke, E. Dufresne, and R. Merlin

Department of Physics, University of Michigan, 500 East University, Ann Arbor, Michigan 48109-1120

(Presented on 22 August 2001)

The short pulses of hard $\mathrm{x}$ rays from synchrotron and laser based sources are sensitive probes of lattice dynamics on an ultrafast time scale. Using pump-probe time-resolved x-ray diffraction, we are able to follow the propagation of a picosecond coherent acoustic pulse in an ultrafast laser-strained single crystal. Comparison of the data with dynamical diffraction simulations allows for the quantitative determination of both the surface and bulk components of the associated strain. This technique is scalable to femtosecond and shorter time scales as x-ray pulses become shorter in duration, such as in fourth generation light sources. In addition, the diffraction of $\mathrm{x}$ rays off of coherent optical phonons may lead to the production of a femtosecond x-ray switch. (C) 2002 American Institute of Physics. [DOI: 10.1063/1.1448147]

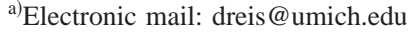

